\title{
G

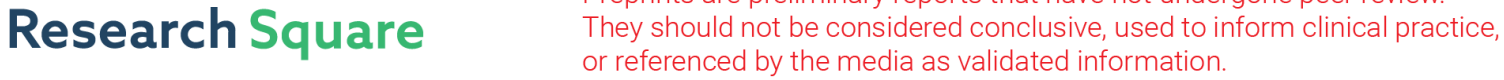 \\ Clinical Study of THSD7A, SOD2 and AR Antigens in Idiopathic Membranous Nephropathy
}

\section{Honggang Wang}

Department of Nephrology, The Second Hospital, Cheeloo College of Medicine, Shandong University, Jinan, Shandong

\section{Tingting Zhao}

Department of Respiratory and Critical Care Medicine, Shandong Provincial Chest Hospital Affiliated to Shandong University, Jinan, Shandong

\section{Jianwei Dong}

Department of Thoracic surgery, The people's Hospital of Rongcheng, Rongcheng, Shandong

\section{Yan Zhou}

Department of Nephrology, Shandong Provincial Hospital, Cheeloo College of Medicine, Shandong University, Jinan, Shandong

\section{Jing Huang}

Department of Nephrology, Jinan Shizhong People's Hospital, Jinan, Shandong

\section{Caifeng Gao}

Department of Nephrology, Jinan Shizhong People's Hospital, Jinan, Shandong

\section{Lei Chen}

Department of Nephrology, Jinan Shizhong People's Hospital, Jinan, Shandong

\section{Jiatong Li}

Department of Nephrology, Shandong Provincial Hospital Affiliated to Shandong First Medical University \& Shandong Academy of Medical Sciences, Jinan, Shandong

\section{Rong Wang}

Department of Nephrology, Shandong Provincial Hospital, Cheeloo College of Medicine, Shandong University, Jinan, Shandong

\section{Bing Chen ( $\nabla$ chenbing3668@163.com )}

Department of Nephrology, Shandong Provincial Hospital, Cheeloo College of Medicine, Shandong University, Jinan, Shandong

\section{Research Article}

Keywords: Idiopathic membranous nephropathy, THSD7A, SOD2, AR, tumor

Posted Date: December 8th, 2021 
DOI: https://doi.org/10.21203/rs.3.rs-1054956/v1

License: (c) (1) This work is licensed under a Creative Commons Attribution 4.0 International License. Read Full License 


\section{Abstract \\ Background}

To explore the expression of THSD7A, SOD2 and AR podocyte antigens in the renal tissue of patients with idiopathic membranous nephropathy (IMN) and its clinical diagnostic value in IMN.

\section{Method}

This study retrospectively collected 150 renal tissue specimens and clinical data of patients undergoing renal biopsy in the Department of Nephrology, Shandong Provincial Hospital, including 130 IMN and 20 non-IMN patients. We use immunohistochemical staining to detect the expression of THSD7A, SOD2, AR antigens in the kidney tissue of patients. We focus on analyzing the clinical and pathological characteristics of IMN patients with positive THSD7A antigen expression, and analyze the prognosis of THSD7A-IMN patients and tumor occurrence. Meanwhile, the sensitivity and specificity of those antigens for the diagnosis of IMN were discussed.

\section{Results}

There were only 6 patients with THSD7A positive expression among 150 patients with glomerular disease, of which 5 patients were all IMN patients, and the patients' serum anti-PLA2R antibodies(antiPLA2R-ab) were all negative. The positive rate of THSD7A in IMN was 3.85\%, and the positive rate in IMN patients with anti-PLA2R-ab negative was $5.50 \%$. Meanwhile, the sensitivity and specificity of renal tissue THSD7A in diagnosing IMN is $4.0 \%$ and $95 \%$. In addition, the IgG subtype of THSD7A-IMN was mainly IgG4; Among them, 2 patients with IMN had both THSD7A and PLA2R antigen staining. This study also suggests that there is no significant difference in clinical and pathological characteristics between THSD7A positive and negative group. The follow-up of THSD7A-IMN patients found no tumors. This study also showed that there were 11 cases of anti-PLA2R-ab negative IMN patients with SOD2 antigen expression (8.56\%), 10 cases of anti-PLA2R-ab negative and 4 cases of anti-PLA2R-ab positive IMN patients with AR antigen expression (10.77\%), but no obvious SOD2 and AR antigen expression in SMN and other glomerular diseases.

\section{Conclusions}

Glomerular THSD7A antigen has a certain expression rate and high specificity in IMN, but its expression rate is low, and it can be used as a specific diagnostic index for IMN supplement. Kidney tissue SOD2, AR podocyte target antigens have a low positive rate in IMN, and further research is needed to evaluate the condition of IMN. 


\section{Introduction}

Membranous nephropathy ( $\mathrm{MN}$ ) is the most common pathological type of adult nephrotic syndrome and is also a common cause of end-stage renal disease (ESRD) ${ }^{1}$. At present, IMN is considered to be a specific autoimmune kidney disease mediated by anti-podocyte antibodies. In 2009, Beck et al. ${ }^{2}$ found that PLA2R is the specific target antigen of most cases of IMN, and anti-PLA2R antibodies have become an important serological indicator for IMN diagnosis and disease monitoring. Nonetheless, approximately $30 \%$ of IMN patients are clinically negative for serum anti-PLA2R antibodies. Although the etiology remains unclear, it is speculated that other undetected endogenous glomerular target antigens exist. Recently, novel podocyte antigens, such as THSD7A, SOD2 and AR, have been discovered, providing a new molecular biological basis for the pathological mechanism of the molecular damage caused by IMN immune complex deposition. In 2014, Tomas et al. ${ }^{3}$ detected anti-glomerulus antibodies, namely, antiTHSD7A antibodies, in serum samples of IMN patients negative for anti-PLA2R-ab. Hence, THSD7A may constitute a new indicator for IMN, with higher specificity for disease diagnosis and activity monitoring, especially in IMN patients negative for anti-PLA2R-ab. In addition to PLA2R and THSD7A, other related podocyte cytoplasmic pathogenic antigens have been reported, including superoxide dismutase (SOD2) and aldose reductase (AR). In 2010, Prunotto et al. ${ }^{4}$ examined the serum of IMN patients and found the presence of anti-AR and SOD2 antibodies. Additionally, electron microscopy showed co-deposition of anti-AR and anti-SOD2 IgG4 and C5b-9 in podocytes. These findings suggest that expression of THSD7A, SOD2, and AR in podocytes and the formation of antibodies may play an important role in the initiation and progression of IMN. Therefore, further research to clarify coexisting antigens in the podocyte membrane as well as subepithelial deposits other than PLA2R is essential for understanding the pathogenesis of IMN.

Much research on the diagnosis and treatment value of IMN has been carried out, including investigations of serum anti-PLA2R antibodies, renal tissue PLA2R expression and IgG4 deposition. We also previously conducted in-depth analysis of anti-PLA2R antibodies, confirming it is an important biomarker for the diagnosis of IMN. Furthermore, the level of antibody is closely related to disease activity. Our study of patients negatives for the anti-PLA2R antibody demonstrated that combined kidney tissue PLA2R and IgG4 staining can effectively improve the accuracy of disease diagnosis ${ }^{5}$.

Nevertheless, there are few reports and a lack of in-depth research on related podocyte antigens, such as THSD7A, SOD2 and AR, in China. Accordingly, this study aims to further investigate expression of these antigens in the renal tissues of patients with glomerular diseases, especially IMN patients who are antiPLA2R-ab negative, to explore the role of podocyte-associated antigens in the pathogenesis of IMN. The goal is to further reveal the pathogenesis of IMN and to provide a basis for guiding clinical treatment and observation of prognosis.

\section{Materials And Methods}


Research object: For this study, 150 patients diagnosed with glomerular disease by renal biopsy with complete clinical data from January 2015 to December 2019 in the Department of Nephrology, Shandong Provincial Hospital were selected. The patients were divided into an IMN group (130 cases) and a nonIMN group (20 cases). IMN was further divided into an antibody-negative group (91 cases) and an antibody-positive group (40 cases) according to serum anti-PLA2R-ab expression. Non-IMN included 5 cases, diagnosed as MCD, IgA nephropathy, lupus nephritis and FSGS. The diagnostic criteria for IMN are based on the results of renal biopsy while eliminating secondary clinical factors such as autoimmune diseases, tumors, drugs, heavy metals, human immunodeficiency virus infection and other factors. The patients were not given hormone and immunosuppressive therapy before biopsy and were followed up for more than 6 months. The IMN treatment remission standard adopted that established by the "Globular Nephritis Clinical Practice Guidelines" published by KDIGO in 2012, as follows. Complete remission (CR) was defined as 24 hUpro $<0.3 \mathrm{~g}$ on the premise of stable renal function. Partial remission (PR) was defined as 24 hUpro decreased by $50 \%$ on the premise of stable renal function and less than $3.5 \mathrm{~g}$ on the premise of stable renal function. No remission indicated that the above remission criteria were not met. Serious complications during follow-up included death or severe pulmonary infection, cerebral infarction and tumor. This study was approved by the Medical Ethics Committee of Shandong Provincial Hospital (LCYJ: no. 2019-105) and followed ethical standards. This study has obtained verbal consent from patients.

\section{Clinical and laboratory data collection}

We collected general clinical data for the patients, including age, sex, disease course, and blood pressure at admission. Laboratory examination data included routine blood tests (white blood cells, hemoglobin, platelets), routine urine tests (urinary protein, urine red blood cells, etc.), 24-hour urine protein quantification, liver function biochemistry (albumin (ALB), alanine aminotransferase (ALT), aspartate aminotransferase (AST), $\beta 2$ microglobulin (BMG), serum creatinine (CREA), blood urea nitrogen (BUN), eGFR, total cholesterol (CHOL), low-density lipoprotein (LDL-C), high-density lipoprotein (HDL-C), glucose (GLU), etc.), immunological indicators (Ig, C3C4, anti-PLA2R-ab, anti-nuclear antibody, ANCA antibody, etc.) and infection indicators (virus series, ESR, etc.). eGFR was calculated based on the Modification of Diet in Renal Disease (MDRD) formula.

\section{Pathological data}

Percutaneous renal puncture was used to collect kidney tissue specimens under the guidance of B ultrasound, and the renal puncture tissue was stained for light and electron microscopy examination. Membranous nephropathy is divided into 4 stages. If two stages occur at the same time, the high stage was the final stage; stage III and stage IV were defined as advanced pathological stages. We then observed glomeruli and determined the presence of focal segmental sclerosis, glomerular sclerosis, mesangial proliferation disease, crescent, balloon adhesion, endothelial hyperplasia, acute and chronic tubular disease, inflammatory cell infiltration, and small blood vessel disease, among others.

\section{Anti-PLA2R detection method}


The anti-phospholipase A2 receptor antibody IgG detection kit (enzyme-linked immunosorbent assay) produced by EUROIMMUN (product no. EA 1254-9601 G) was used and performed according to the standard procedure in the manual. The normal reference range was $0-20 \mathrm{RU} / \mathrm{ml}$.

Kidney tissue IgG4, PLA2R, THSD7A, SOD2, AR antigen detection: We collected kidney biopsy paraffin sections obtained from patients by renal puncture and used immunohistochemistry to detect expression of target antigens and IgG4 in the kidney tissue. An anti-THSD7A antibody was purchased from Novus and used at a dilution ratio of 1:1000. Antibodies against SOD2 and AR were purchased from Abcam and used at dilution ratios of $1: 100$ and 1:200, respectively.

\section{Statistical methods}

All data in this study were analyzed using SPSS 22.0. Measurement data are generally described by $\mathrm{x} \pm \mathrm{s}$. Measurement data were tested and analyzed before applying a normality test and analysis of variance. The independent sample t test or analysis of variance was employed for statistical analysis between measurement data groups that satisfied the normal distribution, and the variance was uniform. Nonnormally distributed data are represented by $M(1 / 4,3 / 4)$. The rank sum test was used for comparisons between groups; categorical variables are represented by a rate or percentage, and the chisquared test was used for comparisons between groups.

\section{Results}

\section{Expression and distribution of each podocyte antigen in the kidney tissue of patients with glomerular disease}

In this study, there were 6 THSD7A-positive patients among 150 with glomerular disease; 5 of them had IMN. The serum of these patients was negative for anti-PLA2R-ab, and their kidney pathology revealed dark-brown material capillary loops and basement membranes with granular or linear deposits. The rate of THSD7A antigen positivity in IMN was 3.85\%, and that in IMN patients who were anti-PLA2R-ab negative was $5.50 \%$. THSD7A was also positive in the renal tissue of one patient with lupus nephritis (WHO V), though no significant expression of THSD7A was found in anti-PLA2R-ab-positive IMN or other glomerular diseases. It total, 11 anti-PLA2R-ab-negative IMN patients were positive for SOD2 antigen expression, and 10 anti-PLA2R-ab-negative IMN patients and 4 antibody-positive IMN patients were positive for AR antigen expression. Expression of these antigens was not observed in SMN or other glomerular diseases.

We focused on the clinical efficacy of expression of THSD7A, SOD2 and AR in renal tissues for diagnosing IMN. The sensitivity of renal tissue THSD7A for diagnosing IMN was $4.0 \%$, and the specificity reached $95 \%$. The rates of AR and SOD2 positivity were $10.76 \%$ and $8.46 \%$, respectively. The negative likelihood ratios of SOD2 and AR for the diagnosis of IMN were 1.12 and 1.16, respectively, both greater 
than 0.1. The above results indicate that expression of these two antigens in IMN was low and that the significance of excluding IMN was limited.

In this study, we further detected expression of PLA2R and IgG4 in the kidney tissues of THSD7A-, SOD2-, and AR-positive IMN patients, and the results showed that the anti-THSD7A IgG subtype in IMN was mainly IgG4 (4/5). In addition, the kidney tissues of two patients stained positively for THSD7A and PLA2R antigens at the same time. In previous studies, PLA2R and THSD7A were found to be mutually exclusive; that is, the anti-THSD7A antibody and THSD7A antigen are only present in PLA2R-negative IMN patients. In IMN with positive expression of SOD2 and AR, pathological kidney changes mainly involved IgG4 deposition. The specific results are provided in Table 1 and Figure 1.

Table 1

The expression and distribution of each antigen in IMN and non-IMN kidney tissues and the expression of IgG4/PLA2R

\begin{tabular}{|llll|}
\hline & THSD7A & SOD2 & AR \\
\hline Anti-PLA2R-ab (-) IMN & 5 & 11 & 10 \\
\hline $\operatorname{IgG4}(+) / P L A 2 R(+)$ & 2 & 8 & 6 \\
\hline $\operatorname{lgG} 4(+) / P L A 2 R(-)$ & 2 & 1 & 2 \\
\hline $\operatorname{lgG} 4(-) / P L A 2 R(-)$ & 1 & 2 & 2 \\
\hline Anti-PLA2R-ab (+) IMN & 0 & 0 & 4 \\
\hline No-IMN & 1 & 0 & 0 \\
\hline
\end{tabular}

\section{Comparison of clinical and pathological characteristics of IMN patients with THSD7A-positive and -negative in kidney tissue}

The patients were divided into THSD7A+ (5 cases) and THSD7A- (125 cases) groups according to expression of THSD7A in kidney tissues, and the clinicopathological characteristics of the two groups were analyzed to understand the clinical and pathological characteristics of THSD7A+ IMN (Table 2). The THSD7A- IMN patients were mostly male (male to female ratio 4:1), and the eGFR of the positive group was lower than that of the negative group (82.20 \pm 26.08 VS 103.43 \pm 22.76$)$, with a significant difference between the groups $(P<0.05)$. In contrast, no significance was found for other related clinical indicators and pathological changes. The results of our study suggest that there is no significant difference in clinical and pathological characteristics between THSD7A-positive and THSD7A-negative IMN patients. 
Table 2

Comparison of clinical and pathological characteristics of THSD7A positive and negative IMN patients

\begin{tabular}{|c|c|c|c|}
\hline & $\begin{array}{l}\text { THSD7A+ } \\
(\mathrm{N}=5)\end{array}$ & $\begin{array}{l}\text { THSD7A- } \\
(\mathrm{N}=125)\end{array}$ & $p$ \\
\hline gender(male/female) & $4 / 1$ & $74 / 51$ & 0.352 \\
\hline age & $51.20 \pm 10.03$ & $45.74 \pm 13.76$ & 0.382 \\
\hline 24hUpro & $5.03 \pm 1.35$ & $4.80 \pm 2.62$ & 0.842 \\
\hline HGB & $136.2 \pm 17.98$ & $137.64 \pm 23.63$ & 0.893 \\
\hline WBC & $6.11 \pm 1.06$ & $8.48 \pm 14.66$ & 0.719 \\
\hline PLT & $268.60 \pm 40.74$ & $278.56 \pm 71.99$ & 0.760 \\
\hline AST & $30.00 \pm 13.84$ & $23.92 \pm 11.72$ & 0.261 \\
\hline ALT & $28.40 \pm 14.17$ & $24.44 \pm 17.79$ & 0.624 \\
\hline ALB & $24.50 \pm 2.91$ & $26.21 \pm 6.17$ & 0.537 \\
\hline TG & $2.07 \pm 0.53$ & $2.40 \pm 1.24$ & 0.562 \\
\hline $\mathrm{CHOL}$ & $6.83 \pm 3.62$ & $8.41 \pm 2.58$ & 0.189 \\
\hline HDL-C & $1.49 \pm 0.46$ & $1.58 \pm 0.53$ & 0.735 \\
\hline LDL-C & $4.29 \pm 2.37$ & $5.33 \pm 2.00$ & 0.259 \\
\hline BUN & $7.42 \pm 4.80$ & $5.74 \pm 6.07$ & 0.543 \\
\hline CREA & $69.56 \pm 26.38$ & $67.27 \pm 30.96$ & 0.871 \\
\hline eGFR & $82.20 \pm 26.08$ & $103.43 \pm 22.76$ & $0.045^{\star}$ \\
\hline BMG & $1.85 \pm 0.41$ & $2.33 \pm 1.18$ & 0.362 \\
\hline $\lg G$ & $9.14 \pm 2.23$ & $6.84 \pm 2.79$ & 0.072 \\
\hline $\operatorname{lgG} 4$ & $468.20 \pm 98.33$ & $381.86 \pm 366.34$ & 0.601 \\
\hline $\operatorname{lgM}$ & $0.97 \pm 0.52$ & $1.15 \pm 0.52$ & 0.461 \\
\hline C3 & $1.20 \pm 0.20$ & $1.18 \pm 0.19$ & 0.940 \\
\hline C4 & $0.28 \pm 0.74$ & $0.31 \pm 0.19$ & 0.757 \\
\hline Pathological features & $4 / 5(80.00 \%)$ & $68 / 125(54.4 \%)$ & 0.259 \\
\hline \multicolumn{4}{|c|}{$\begin{array}{l}\text { Note: 24hUpro, 24-hour urine protein quantification; HGB, Hemoglobin; WBC, white blood cell; PLT, } \\
\text { Platelets; AST, aspartate aminotransferase; ALT, alanine aminotransferase; ALB, albumin; TG, } \\
\text { Triglycerides; CHOL, total cholesterol; HDL-C, high-density lipoprotein; LDL-C, low-density lipoprotein; } \\
\text { BUN, blood urea nitrogen; CREA, serum creatinine; eGFR, Glomerular filtration rate; BMG, } \beta 2 \\
\text { macroglobulin; IgG4, immunoglobulin }\end{array}$} \\
\hline \multicolumn{4}{|l|}{$P *: P<0.05$} \\
\hline
\end{tabular}




\begin{tabular}{|c|c|c|c|}
\hline & $\begin{array}{l}\text { THSD7A+ } \\
(\mathrm{N}=5)\end{array}$ & $\begin{array}{l}\text { THSD7A- } \\
(\mathrm{N}=125)\end{array}$ & \\
\hline Spherical hardening & $3 / 5(60.00 \%)$ & $43 / 125(34.40 \%)$ & 0.240 \\
\hline Mesangial hyperplasia & $2 / 5(40.00 \%)$ & 15/125(12.00\%) & 0.069 \\
\hline Acute renal tubular disease & $2 / 5(40.00 \%)$ & 15/125(12.00\%) & 0.069 \\
\hline Chronic renal tubular disease & $1 / 5(20.00 \%)$ & $14 / 125(11.20 \%)$ & 0.546 \\
\hline Inflammatory cell infiltration & $3 / 5(60.00 \%)$ & $51 / 125(40.80 \%)$ & 0.149 \\
\hline Vascular proliferative changes & $1 / 5(20.00 \%)$ & $1 / 125(0.8 \%)$ & 0.076 \\
\hline Crescent & 0 & $3 / 125(2.40 \%)$ & 0.640 \\
\hline Endothelial hyperplasia & $1 / 5(20.00 \%)$ & 16/125(12.80\%) & \\
\hline \multicolumn{4}{|l|}{ Balloon adhesions } \\
\hline \multicolumn{4}{|c|}{$\begin{array}{l}\text { Note: 24hUpro, 24-hour urine protein quantification; HGB, Hemoglobin; WBC, white blood cell; PLT, } \\
\text { Platelets; AST, aspartate aminotransferase; ALT, alanine aminotransferase; ALB, albumin; TG, } \\
\text { Triglycerides; CHOL, total cholesterol; HDL-C, high-density lipoprotein; LDL-C, low-density lipoprotein; } \\
\text { BUN, blood urea nitrogen; CREA, serum creatinine; eGFR, Glomerular filtration rate; BMG, } \beta 2 \\
\text { macroglobulin; IgG4, immunoglobulin }\end{array}$} \\
\hline$P *: P<0.05$ & & & \\
\hline
\end{tabular}

\section{Clinical Data And Follow-up Survey Of Thsd7a-positive Patients}

To further understand the effect of THSD7A on the treatment and prognosis of IMN patients, the general clinical characteristics and treatment prognosis of 5 patients with THSD7A-positive IMN were recorded and followed up; the specific results are presented in Tables 3 and 4. Table 3 shows clinical data for 5 patients with THSD7A-positive IMN. All patients exhibited first clinical manifestations of proteinuria and hypoalbuminemia before treatment. The average ALB level at renal biopsy was $24.50 \pm 2.91 \mathrm{~g} / \mathrm{L}$, and the average CREA level was $69.56 \pm 26.38 \mu \mathrm{mol} / \mathrm{L}$. During follow-up, four patients achieved clinical remission $(80 \%)$, with patients 3,4 , and 5 achieving complete remission (60\%) and patient 1 partial remission (20\%). All 5 THSD7A-positive IMN patients in this study were followed up for more than 6 months, with no tumor development. 
Table 3

Clinical and pathological data of 5 cases of THSD7A-related IMN

\begin{tabular}{|lllllllll|}
\hline number & gender & age & 24hUpro & ALB & BUN & CREA & eGFR & $\begin{array}{c}\text { Tumor } \\
\text { marker }\end{array}$ \\
\hline 1 & Male & 55 & 4.5 & 24 & 5.6 & 47.7 & 108 & - \\
\hline 2 & Male & 39 & 7.0 & 22.7 & 8.4 & 95.1 & 86 & - \\
\hline 3 & Male & 66 & 5.82 & 25.9 & 15.2 & 100 & 70 & - \\
\hline 4 & Male & 61 & 4.7 & 24.9 & 6.2 & 74 & 91 & - \\
\hline 5 & Female & 48 & 4.12 & 28.7 & 2.7 & 44 & 44 & - \\
\hline
\end{tabular}

Table 4

Prognostic follow-up status of 5 cases of THSD7A-related IMN

\begin{tabular}{|lllllll|}
\hline number & treatment & tine & 24hUpro & ALB & CEA & Prognosis/tumor \\
\hline 1 & Pre+CSA & 11 & 1.92 & 37.7 & 60.5 & PR \\
\hline 2 (Urine Chromium+) & Pre+CTX & 7 & 5.03 & 26.9 & 81.3 & not remission \\
\hline 3 & Pre & $>12$ & 0.03 & 45 & 66 & $\mathrm{CR}$ \\
\hline 4 & Pre+CSA & $>12$ & 0.02 & 42.1 & 86.3 & $\mathrm{CR}$ \\
\hline 5 & Pre+FK506 & 12 & 0.29 & 35.1 & 41.5 & $\mathrm{CR}$ \\
\hline $\begin{array}{l}\text { Note:24hUpro,24-hour urine protein quantification; ALB, albumin; CREA, Creatinine; eGFR, Glomerular } \\
\text { filtration rate; Pre, hormone;CAS, Cyclosporine; CTX, CyclophosphamidelPR, Partial Remission; CR, } \\
\text { Complete Remission. }\end{array}$
\end{tabular}

\section{Discussion}

\section{Clinical Study Of Thsd7a-positive Imn}

THSD7A is the second pathogenic target antigen expressed on podocytes and in addition to PLA2R, is involved in the pathogenesis of IMN. Presently, the main methods for clinical detection of THSD7A include renal tissue immunostaining and serological antibody analysis. Studies have found that the sensitivity and specificity of renal biopsy tissue staining for THSD7A are higher than those of serological detection; furthermore, recombinant THSD7A can be recognized from glomerular decomposition or immunoprecipitation in all serologically positive samples ${ }^{3,6}$. However, some studies have found no significant difference for the two detection methods in the rate of THSD7A positivity, and both can be used to guide the diagnosis and treatment of diseases; immunohistochemistry does remain the most effective detection method for diagnosis ${ }^{7}$. 
In this study, positive expression rate of THSD7A was 3.85\% in IMN, and all positive cases involved antiPLA2R-ab negative IMN; in contrast, the rate of THSD7A positivity in PLA2R-negative IMN was $5.50 \%$. Moreover, no significant THSD7A expression was found in anti-PLA2R antibody-positive IMN and other glomerular diseases. The sensitivity of kidney tissue THSD7A antigen detection for the diagnosis of IMN was $4.0 \%$, and the specificity was $95 \%$. Overall, THSD7A may be used as a supplementary indicator for the diagnosis of PLA2R negativity. Although the results of this study are basically consistent with related reports, there are some differences. For example, Tomas et al. ${ }^{3}$ reported a rate of THSD7A positivity in the glomeruli of IMN patients in Europe and the United States of $2.5 \%$ 5.0\%, which is equivalent to $8 \%-14 \%$ for anti-PLA2R-ab negative IMN. In addition, Iwakura et al. ${ }^{8}$ found that the rate of THSD7A positivity in the glomeruli of Japanese patients was $9.1 \%$, accounting for $19.1 \%$ of non-PLA2R-IMN. Conversely, research in China ${ }^{9}$ suggested a glomerular THSD7A-positive rate of only approximately $2 \%$. The reasons for differences may be related to various factors, such as geographic, ethnic or genetic differences. Moreover, the proportion of THSD7A as a pathogenic antigen is small, kidney biopsy is usually performed while the patient is being treated, and the timing at different stages of the disease course and other factors vary ${ }^{10}$. Previous studies have shown that the prevalence of THSD7A is relatively low and that it is mainly expressed in PLA2R-negative cases; thus, it can be used as a complement to IMN-specific diagnostic indicators. Furthermore, THSD7A has not been detected thus far in healthy people or in those with glomerular diseases other than IMN. In addition, the rate of THSD7A positivity in secondary membranous nephropathy (SMN) is extremely low. For instance, only 2 of 157 SMN patients tested for THSD7A in the study by Ren et al. " were found to have anti-THSD7A antibodies in their serum. In another study, only 1 of 114 patients with SMN had anti-THSD7A antibodies. Therefore, THSD7A can be used for differential diagnosis of IMN and other glomerular diseases, including SMN.

In this study, 5 patients had THSD7A-positive IMN, and we performed renal tissue IgG4/PLA2R antigen staining for these cases. The results showed that IgG4 was the main anti-THSD7A IgG subtype in IMN $(4 / 5)$, which is essentially consistent with the relevant literature. Many studies have confirmed that the IgG4 subtype is dominant in IMN, and IgG4 is the main antibody component of IMN-related target antigen (PLA2R, THSD7A, SOD2, a-ENO, AR) autoantibodies 2,4,11; conversely, IgG1 and IgG3 are mainly involved in SMN (such as lupus nephritis) ${ }^{3,12}$. In this study, 2 IMN patients were also positive for both THSD7A and PLA2R expression in kidney tissues. On the one hand, this finding indicates that PLA2R antigen detection in kidney tissue is sensitive and specific for the diagnosis of IMN and can be used as a supplementary detection method for patients with anti-PLA2R antibody negativity and those who have undergone renal needle biopsy. On the other hand, co-expression of THSD7A and PLA2R in kidney tissue has been proven. The current consensus in the literature is that THSD7A mainly exists in patients who are PLA2R negative. Indeed, it is believed that THSD7A and PLA2R are mutually exclusive. Nevertheless, studies have reported cases with positivity for both antibodies, suggesting that they are not mutually exclusive [14.15]. We consider that when the antigen is exposed, one of the antibodies is an opportunistic antibody. Another possibility is that genetic susceptibility in IMN is related to the HLA-DQAl gene, with the immune system of some individuals being more prone to producing multiple pathogenic antibodies.

Page $11 / 19$ 
Overall, combined detection of THSD7A and PLA2R in the serum and kidney tissue will enhance the accuracy of IMN diagnosis and avoid missed diagnosis.

Additionally, previous studies have found that THSD7A-positive IMN is associated with a high tumor risk ${ }^{11,13,14}$. Wang Tao et al. ${ }^{13}$ also provided clear evidence that THSD7A can be used as a tumor marker for $\mathrm{MN}$ caused by malignant tumors. A tumor-released antigen induces the production of corresponding antibodies, which bind to THSD7A on the glomerulus, causing immune complex deposition in the glomerular podocyte and MN. Regardless, as there are also reports that the incidence of THSD7A-related MN combined with malignant tumors is very low $(2 \%)^{14}$, the chance of THSD7A and malignant tumors cannot be ruled out. In the present study, IMN patients did not develop tumors during follow-up, and the patients were not screened for tumor markers during hospitalization. Moreover, imaging examinations did not detect suspicious tumor-occupying manifestations. Because only 5 patients (a small sample size) were followed up, a larger sample size and longer follow-up time are needed to assess the relationship between THSD7A and tumors. Nevertheless, the age of onset of $M N$ is relatively late, and the probability of malignant tumors emerging is relatively high. Clinically, patients with THSD7A-positive IMN need to be carefully screened for tumors. Early detection and treatment of benign tumors may ameliorate (or even eradicate) the symptoms of nephrotic syndrome.

\section{Relationship between SOD2 and AR podocyte target antigens and IMN}

Regarding the expression and clinical significance of the cytoplasmic antigens SOD2 and AR in IMN kidney tissue, AR and SOD2 only exist on renal tubular epithelial cells in the cortex and medulla under normal circumstances and are not expressed in the glomerulus ${ }^{15,16}$. As an antioxidant metalloenzyme, SOD2 plays an important role in oxidative stress (OS) and antioxidative stress: SOD2 scavenges free radicals to protect against the damage caused to cells. OS is related to the occurrence of many diseases, including kidney diseases. Previous literature on Heymann nephritis, a classic animal model of $\mathrm{MN}^{17}$, confirmed that glomerular damage can be mediated by oxidative free radicals produced by podocytes in the presence of C5b-9. As an antioxidant metalloenzyme, SOD2 reduces the production of urine protein with a protective effect during OS. Additionally, SOD2 expression was significantly enhanced in IMN patients in IMN patients compared with SMN and MCD patients, confirming specific SOD2 glomerular expression in IMN. We speculate that in IMN, cells incur mitochondrial damage, with a large amount of SOD2 released from the mitochondria; thus, SOD2 can be found in IMN renal tissues. Aldose reductase $(A R)$ is involved in the anti-inflammatory and oxidative stress response. Overall, the significance of AR and SOD 2 in the diagnosis and prediction of IMN remain unclear. Some scholars ${ }^{4}$ have detected AR and SOD2 in kidney biopsy specimens from patients with IMN. Anti-AR and SOD2 IgG4 and C5b-9 colocalize in the electronic compact of podocytes, and it is speculated that SOD2 and AR may be involved in the pathogenesis of IMN as pathogenic antigens. The study proposed that AR and SOD2 are novel proteins expressed in glomeruli in IMN that are recognized by specific circulating antibodies and that these antibodies can be detected in glomerular subepithelial immune deposits. AR and SOD2 should be considered glomerular antigens related to the immune process.

Page $12 / 19$ 
In this study, the rates of SOD2 and AR positivity in IMN were relatively low, with no significant expression in SMN or other glomerular diseases. We also found that the antibody subtypes for SOD2 and AR-IMN are consistent with THSD7A-positive IMN, with both being dominated by IgG4. The study found that the positive rates for the two antigens were low; the negative likelihood ratios of these two antigens were both $<1$. Nonetheless, due to the small number of non-IMN samples used, the specificity of SOD2 and AR in the diagnosis of IMN need further analysis. The above results indicate that the significance of SOD2 and $A R$ in the diagnosis and exclusion of IMN is limited. Because of the biological characteristics of SOD2, anti-SOD2 antibody expression may occur when the body is affected by autoimmune diseases or liver and kidney damage, among others. Therefore, SOD2 cannot specifically diagnose IMN: the antiSOD2 antibody should not be used as the sole diagnostic and therapeutic index for IMN. However, there are reports suggesting that SOD2 levels are related to urine protein. A study of anti-PLA2R and anti-SOD2 antibodies in China reported that patients negative for these antibodies had higher levels of ALB and eGFR, lower levels of CREA and a shorter time to CR. In general, expression of AR can be significantly enhanced when under inflammatory stimulation or oxidative stress, modulating the inflammatory response process. In IMN patients, anti-AR antibodies may have been positive due to oxidative stress caused by the disease state. Similar to the anti-SOD2 antibody, we cannot clarify the specific effect of anti-AR antibodies on MN patients; the significance of AR and SOD2 in the diagnosis and prediction of IMN is still unclear, and further basic clinical research is needed.

In this study, we did not detect serum anti-THSD7A, -SOD2 and -AR antibodies but rather assessed expression of antigens in renal tissues and analyzed the relationship between antigens and IMN by immunohistochemistry to provide a reference for clinical diagnosis and treatment. Previous studies have confirmed that tissue staining for renal biopsy antigens is more sensitive and specific than serological examinations. In general, if THSD7A, SOD2 and AR are to be used as biomarkers in clinical applications, they need to be combined with serum antibody detection, and further study of the relationship between serum antibodies and IMN is required. In addition, the non-IMN sample in this study was small, as was the number of THSD7A-, SOD2- and AR-positive cases, imposing certain limitations on clinical and followup results. Larger-sample analysis is needed for more accurate and representative results, such that we can conduct clinical comparative analysis. In addition, for THSD7A-positive patients, we need to strengthen follow-up and the detection of positive tumor markers for an in-depth analysis and discussion on the pathogenesis of IMN to better guide clinical decisions.

Since the discovery of PLA2R in IMN 10 years ago, serological assessment of glomerular disease by nephrologists has become more detailed and accurate with the ongoing exploration of the pathogenesis of glomerular disease. In addition to the antigens related to IMN podocytes evaluated herein, other relevant antigens have been recognized due to the rapid development of glomerular laser microcleavage technology and mass spectrometry for dissolution and digestion of proteins, including four "neoantigens" 18, neuroepidermal growth factor-like protein 1 (Nell-1) and semaphorin 3B in IMN, exostosins 1/2 (exosin 1/2) and NCAM 1, which are associated with the development of lupus nephritis. In fact, approximately $8 \%-16 \%$ of the clinical incidence of IMN is related to the podocyte antigen Nell-1, and related pathological 
features are mainly segmental immune complex deposition, similar to those of THSD7A; moreover, tumors occur in approximately $30 \%$ of patients with Nell-1-associated IMN. Overall, Nell-1 is an important autoantigen recently found to be closely related to the pathogenesis of IMN ${ }^{19}$. Semaphorin $3 B$ (signaling 3B)-associated IMN usually occurs in children under 2 years of age and is generally the main target antigen for pediatric IMN pathogenesis, accounting for approximately $16 \%$ of nonlupus MN in children 20 . In addition, exostosins $1 / 2$ and NCAM 1 have been associated with $30 \%$ and $6 \%$ of lupus nephritis, respectively ${ }^{2122}$. The discovery of these new antigens is of great significance for the diagnosis and differential diagnosis of IMN, as well as further study of the pathogenesis of IMN. Such knowledge prompts us to continuously re-examine the pathogenesis, clinical diagnosis and treatment of IMN to achieve more personalized precision medicine for IMN.

\section{Conclusion}

In summary, the results of this study suggest that the glomerular THSD7A antigen is expressed at a certain rate in IMN, with high specificity; its low expression can be used as a supplementary diagnostic index for IMN. Renal tissue SOD2, AR and other related podocyte target antigens have a low rate of positivity in IMN. Overall further IMN research is needed. Further studies with increased sample sizes are warranted, is an extended follow-up period. In-depth research and discussion on the role of HSD7A, SOD2 and $\mathrm{AR}$ in the pathogenesis of MN, especially IMN, are needed to better guide clinical practice.

\section{Abbreviations}

IMN, idiopathic membranous nephropathy; ESRD, end-stage renal disease; SOD2, superoxide dismutase; $A R$, aldose reductase; $C R$, complete remission; PR, Partial remission; ALB, albumin; ALT, alanine aminotransferase; AST, aspartate aminotransferase; BMG, $\beta 2$ macroglobulin; CREA, serum creatinine; BUN, blood urea nitrogen; CHOL, total cholesterol; LDL-C, low-density lipoprotein; HDL-C, high-density lipoprotein.

\section{Declarations}

\section{Ethics approval and consent to participate:}

This study was conducted in accordance with the Declaration of Helsinki. The study protocol was approved by the Ethics Committee of Shandong Provincial Hospital (LCYJ: No. 2019-105).

This study is a retrospective study, which only collects patient-related information. The research risk is not greater than the minimum risk. Therefore, this study does not need to obtain written informed consent from patients, but to follow up patients and their families and obtain oral consent. This study has obtained verbal consent from patients. "Waiver of written informed consent application" was reviewed and approved by the Shandong Provincial Hospital ethics committee, and agree and approve the study. 
The ethical approval document has been issued in the previous period, and the document number is "Provincial Medical Lund Approval (LCYJ: NO. 2019-105)".

Consent for publication: Not applicable.

Availability of data and materials: The datasets used and/or analyzed during the current study will be available from the corresponding author on reasonable request.

Competing interests: All authors declare that they have no competing interests.

Funding: This study was supported by grants from the Primary Research \& Development Plan of Shandong Province (2018GSF118227), the Science and Technology Plan (673 and 741) of Shizhong District of Jinan City, Clinical Medical Science and Technology Development Plan of Jinan City, Shandong Province区202019186『and Horizontal issues of Shandong University(6020121011).

Author's contributions: Honggang Wang, Tingting Zhao, Bing Chen, Rong Wang conceived the study. Honggang Wang, Yan Zhou, Jing Huang, Caifeng Gao, Lei Chen, Jiatong Li led data collection. Honggang Wang, Jianwei Dong did the statistical analyses. Honggang Wang, Tingting Zhao wrote the first draft. All authors critically reviewed the article and approved the final draft.

Acknowledgments: We thank all participants in the survey and all staff members involved in this study for their hard work in data collection.

\section{References}

1. Ronco P, Debiec H. Pathophysiological advances in membranous nephropathy: time for a shift in patient's care. Lancet. May 16 2015;385(9981):1983-92. doi:10.1016/S0140-6736(15)60731-0

2. Beck LH, Bonegio RG, Lambeau G, et al. M-type phospholipase $A 2$ receptor as target antigen in idiopathic membranous nephropathy. N Engl J Med. Jul 02 2009;361(1):11-21. doi:10.1056/NEJMoa0810457

3. Tomas NM, Beck LH, Meyer-Schwesinger C, et al. Thrombospondin type-1 domain-containing 7A in idiopathic membranous nephropathy. N Engl J Med. Dec 11 2014;371(24):2277-2287. doi:10.1056/NEJMoa1409354

4. Prunotto $\mathrm{M}$, Carnevali ML, Candiano $\mathrm{G}$, et al. Autoimmunity in membranous nephropathy targets aldose reductase and SOD2. J Am Soc Nephrol. Mar 2010;21(3):507-19. doi:10.1681/ASN.2008121259

5. Guo W, Zhang Y, Gao C, et al. Retrospective study: clinicopathological features and prognosis of idiopathic membranous nephropathy with seronegative anti-phospholipase A2 receptor antibody. PeerJ. 2020;8:e8650. doi:10.7717/peerj.8650

6. Sharma SG, Larsen CP. Tissue staining for THSD7A in glomeruli correlates with serum antibodies in primary membranous nephropathy: a clinicopathological study. Mod Pathol. 04 2018;31(4):616-622. 
doi:10.1038/modpathol.2017.163

7. Ren S, Wu C, Zhang Y, et al. An update on clinical significance of use of THSD7A in diagnosing idiopathic membranous nephropathy: a systematic review and meta-analysis of THSD7A in IMN. Ren Fail. Nov 2018;40(1):306-313. doi:10.1080/0886022X.2018.1456457

8. Iwakura T, Ohashi N, Kato A, Baba S, Yasuda H. Prevalence of Enhanced Granular Expression of Thrombospondin Type-1 Domain-Containing 7A in the Glomeruli of Japanese Patients with Idiopathic Membranous Nephropathy. PLoS One. 2015;10(9):e0138841. doi:10.1371/journal.pone.0138841

9. Tian C, Li L, Liu T, Qu X, Qiu Y. Circulating antibodies against M-type phospholipase A2 receptor and thrombospondin type-1 domain-containing 7A in Chinese patients with membranous nephropathy. Int Urol Nephrol. Aug 2019;51(8):1371-1377. doi:10.1007/s11255-019-02146

10. Bruschi M, Carnevali ML, Murtas C, et al. Direct characterization of target podocyte antigens and auto-antibodies in human membranous glomerulonephritis: Alfa-enolase and borderline antigens. $J$ Proteomics. Sep 2011;74(10):2008-17. doi:10.1016/j.jprot.2011.05.021

11. Beck LH. PLA2R and THSD7A: Disparate Paths to the Same Disease? J Am Soc Nephrol. Sep 2017;28(9):2579-2589. doi:10.1681/ASN.2017020178

12. Hoxha E, Beck LH, Wiech T, et al. An Indirect Immunofluorescence Method Facilitates Detection of Thrombospondin Type 1 Domain-Containing 7A-Specific Antibodies in Membranous Nephropathy. $J$ Am Soc Nephrol. 02 2017;28(2):520-531. doi:10.1681/ASN.2016010050

13. Wang T, Zhang Y, Liu M, Kang X, Kang L, Zhang H. THSD7A as a marker for paraneoplastic membranous nephropathy. Int Urol Nephrol. Feb 2019;51(2):371-373. doi:10.1007/s11255-01802068-Z

14. Wang J, Cui Z, Lu J, et al. Circulating Antibodies against Thrombospondin Type-I Domain-Containing 7A in Chinese Patients with Idiopathic Membranous Nephropathy. Clin J Am Soc Nephrol. Oct 06 2017;12(10):1642-1651. doi:10.2215/CJN.01460217

15. Barski OA, Papusha VZ, Ivanova MM, Rudman DM, Finegold MJ. Developmental expression and function of aldehyde reductase in proximal tubules of the kidney. Am J Physiol Renal Physiol. Jul 2005;289(1):F200-7. doi:10.1152/ajprenal.00411.2004

16. Terubayashi H, Sato S, Nishimura C, Kador PF, Kinoshita JH. Localization of aldose and aldehyde reductase in the kidney. Kidney Int. Nov 1989;36(5):843-51. doi:10.1038/ki.1989.270

17. HEYMANN W, LUND HZ, HACKEL DB. The nephrotic syndrome in rats; with special reference to the progression of the glomerular lesion and to the use of nephrotoxic sera obtained from ducks. $J L a b$ Clin Med. Feb 1952;39(2):218-24.

18. Ronco P, Plaisier E, Debiec H. Advances in Membranous Nephropathy. J Clin Med. Feb 05 2021;10(4)doi:10.3390/jcm10040607

19. Sethi S, Debiec H, Madden B, et al. Neural epidermal growth factor-like 1 protein (NELL-1) associated membranous nephropathy. Kidney Int. 01 2020;97(1):163-174. doi:10.1016/j.kint.2019.09.014 
20. Sethi S, Debiec H, Madden B, et al. Semaphorin 3B-associated membranous nephropathy is a distinct type of disease predominantly present in pediatric patients. Kidney Int. 11 2020;98(5):1253-1264. doi:10.1016/j.kint.2020.05.030

21. Sethi S, Madden BJ, Debiec H, et al. Exostosin 1/Exostosin 2-Associated Membranous Nephropathy. J Am Soc Nephrol. 06 2019;30(6):1123-1136. doi:10.1681/ASN.2018080852

22. Caza TN, Hassen $\mathrm{SI}$, Kuperman M, et al. Neural cell adhesion molecule 1 is a novel autoantigen in membranous lupus nephritis. Kidney Int. 07 2021;100(1):171-181. doi:10.1016/j.kint.2020.09.016

\section{Figures}



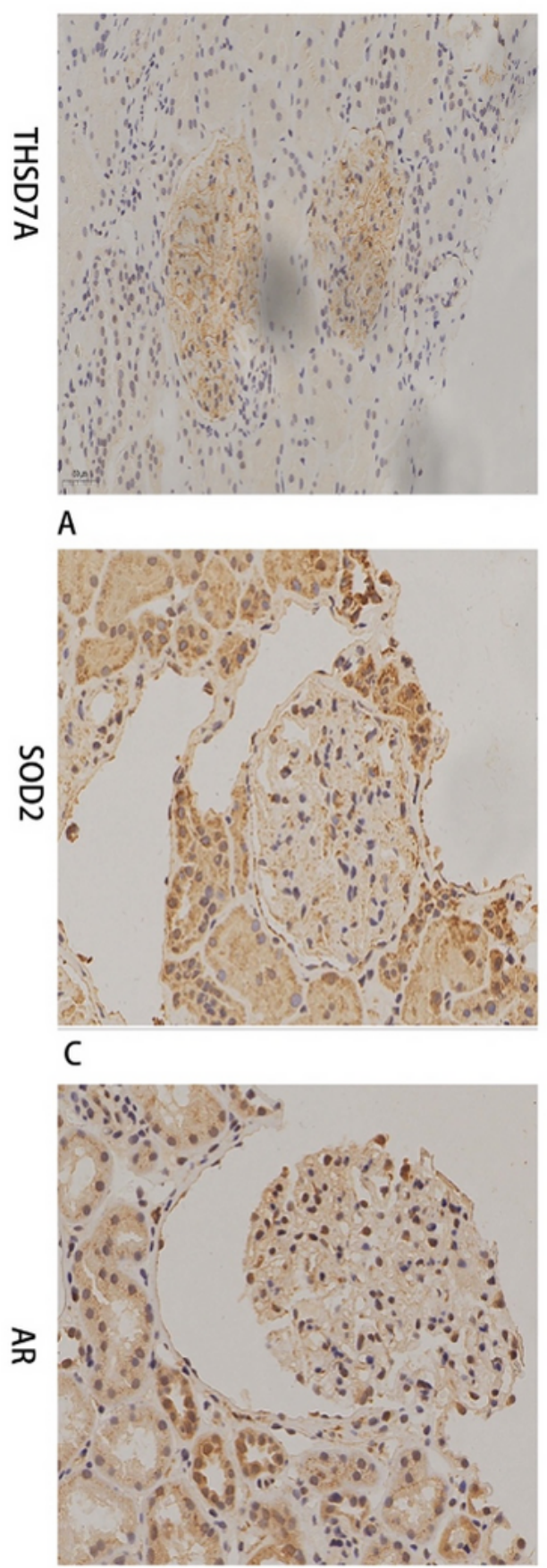

$\mathrm{E}$

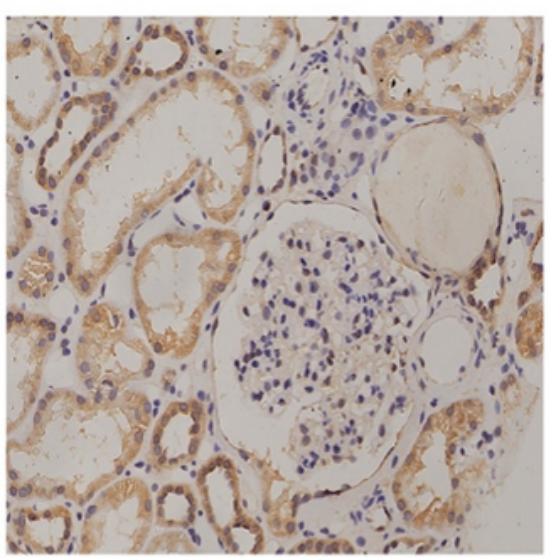

B

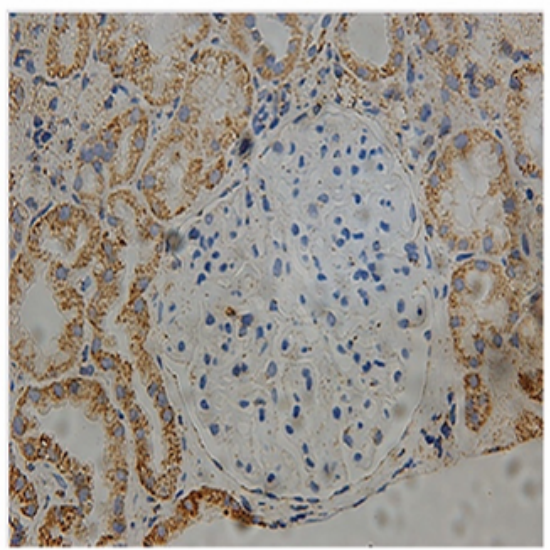

D

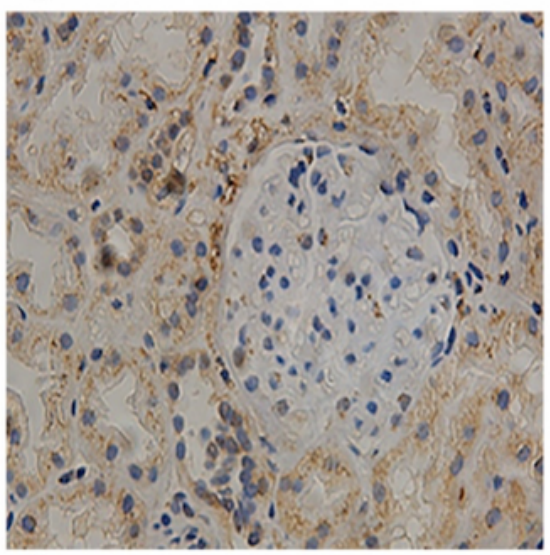

F

\section{Figure 1}

The expression of THSD7A, SOD2 and AR in the kidney tissue of IMN patients Legends for Figure 1: A and $B$ show the co-expression and staining of THSD7A and PLA2R antigens in IMN, that is, THSD7A and PLA2R staining positively in IMN kidney tissue; $C$ shows the positive expression of IgG4 in the kidney tissue of patients with THSD7A-positive IMN; D shows the kidney of patients with IMN Tissue THSD7A staining was negative; $\mathrm{E}$ and $\mathrm{F}$ show the positive and negative expression of SOD2 antigen in the kidney 
tissue of IMN patients, respectively; $\mathrm{G}$ and Figure $\mathrm{H}$ show the positive and negative expression of $A R$ antigen in the kidney tissue of IMN patients, respectively.

\section{Supplementary Files}

This is a list of supplementary files associated with this preprint. Click to download.

- rawdata.xIsx 